\title{
Modelagem e simulação de biorreatores em série para a obtenção de etanol usando a equação de Tosetto
}

\section{Modeling and simulation of serial biorreactors for obtaining ethanol using the Tosetto equation}

Article Info:

Article history: Received 2021-03-25 / Accepted 2021-05-23 / Available online 2021-05-25

doi: $10.18540 /$ jcecvl7iss2pp12228-01-08e

\author{
João Víctor Sales Castro \\ ORCID: https://orcid.org/0000-0002-1651-5506 \\ UFSJ, Brazil \\ E-mail: jvsc55@gmail.com \\ Juan Canellas Bosch Neto \\ ORCID: https://orcid.org/0000-0002-2719-7689 \\ UFSJ, Brazil \\ E-mail: jboschbr@ufsj.edu.br
}

\section{Resumo}

Neste trabalho foi simulado um processo para produção de etanol em regime transiente com biorreatores dispostos em série. A simulação foi realizada no software OCTAVE, resolvendo um sistema de nove equações diferenciais não-lineares, através do método numérico de Runge-Kutta. Com esta simulação foi possível verificar a efetividade da utilização de um biorreator na produção de etanol, considerando as condições de contorno e estabelecendo as características para uma possível otimização da conversão e produtividade no processo. A partir dos resultados da simulação observou-se que a Equação de Tosetto é adequada para a modelagem do processo de obtenção do etanol em biorreatores e condizente com trabalhos publicados. A concentração de células aumentou significativamente, em três horas de processo entrou em regime permanente, que é compatível com a teoria de crescimento celular. Em relação à concentração do substrato foi observado que diminuiu significativamente até 4 horas e após dez horas ocorre um pequeno crescimento na concentração, indicando a inibição pelo substrato. A concentração do produto etanol aumentou de dez por cento a cada passagem pelos reatores 1,2 e 3 até atingir uma concentração de etanol de oitenta gramas por litro em regime transiente. A simulação realizada em três estágios torna o processo mais eficiente ao invés de se utilizar apenas um biorreator.

Palavras-chave: Biorreatores em série. Etanol. Modelagem. Tosetto.

\begin{abstract}
In this work, a process for transient ethanol production was simulated with bioreactors arranged in series. The simulation was performed in the OCTAVE software, solving a system of nine nonlinear differential equations, using the numerical method of Runge-Kutta. With this simulation it was possible to verify the effectiveness of the use of a bioreactor in the production of ethanol, considering the boundary conditions and establishing the characteristics for a possible optimization of the conversion and productivity in the process. From the results of the simulation, it was observed that the Tosetto Equation is suitable for modeling the process of obtaining ethanol in bioreactors and consistent with published works. The concentration of cells increased significantly, in three hours of process it entered a permanent regime, which is compatible with the theory of cell growth. Regarding the concentration of the substrate, it was observed that it decreased significantly up to 4 hours and after 10 hours there is a small increase in concentration, indicating inhibition by the substrate. The concentration of the ethanol product increased by ten percent with each passage through reactors 1,2 and 3 until reaching a concentration of ethanol of eighty grams per liter in a transient regime. The simulation carried out in three stages makes the process more efficient instead of using only one bioreactor.
\end{abstract}


Keywords: Serial bioreactors. Ethanol. Modeling. Tosetto.

\section{Introdução}

Com o desenvolvimento de novas tecnologias, a sociedade se viu dependente de produtos com origem fóssil, em especial os derivados do petróleo, como fonte de energia. Com este aumento pela procura, houve a redução de disponibilidade destes recursos gerando, nas últimas décadas, o aumento de pesquisas na busca de fontes energéticas renováveis alternativas ao petróleo e seus derivados. Com este panorama, surgiu os biocombustíveis, compostos que podem substituir a gasolina no abastecimento automotivo, como por exemplo o etanol e o biodiesel.

Os biocombustíveis tem se tornado uma importante fonte de energia alternativa. São produzidos a partir de fontes renováveis, chamada biomassa, que tem a produção específica para este fim ou a partir de resíduos orgânicos de algum processo, caracterizando a biomassa residual, sendo conhecidas como biocombustíveis de primeira e segunda geração, respectivamente.

$\mathrm{O}$ etanol $\left(\mathrm{C}_{2} \mathrm{H}_{5} \mathrm{OH}\right)$ é um composto orgânico, incolor, volátil, inflamável e totalmente solúvel em água, sendo utilizado mundialmente como combustível automotivo devido a seu elevado índice de octano (108) e bom teor de oxigênio (35\%), o que atribui uma rápida e limpa combustão quando comparado à gasolina (Silva, 2012). Além disso, ele pode ser obtido de três formas: por destilação de líquidos alcoólicos como a cerveja; por via sintética, a partir da hidratação do etileno e etino ou a partir de gases de petróleo e hulha; e por via fermentativa através da fermentação de açúcares (glicose, sacarose e frutose) sob condições anaeróbias (Souza, 2016).

Para essa fermentação, é necessário que as leveduras atuem sobre os açúcares fermentáveis contidos na solução extraída da matéria prima. Contudo, a fermentação alcoólica envolve cerca de doze reações enzimáticas, que sofre ações de diversos fatores como nutrientes, minerais, vitaminas, inibidores, $\mathrm{pH}$, temperatura e afins, que podem ou não favorecer o desempenho das leveduras (Lima; Basso; Amorim, 2001).

Nas últimas décadas, o Brasil se destacou como maior produtor de etanol por via fermentativa em razão do investimento em técnicas de produção industrial. Apesar disso, uma grande parte de suas unidades produtoras, ainda, apresenta possibilidade de melhoras tanto de projeto quanto operacionais (Andrietta et al., 1994).

A modelagem matemática é a área do conhecimento que estuda maneiras de desenvolver e implementar modelos matemáticos de sistemas reais (Aguirre, 2004). Pode ser definida também como uma alternativa de representação, por equações matemáticas, para os balanços de massa de cada componente do biorreator.

Uma vez que se obtém o modelo matemático, é necessário que haja uma verificação do comportamento correto equivalente das equações sob a perspectiva real do sistema. A simulação foi obtida através da resolução de um sistema de equações diferenciais resolvido pelo método numérico de Runge-Kutta. O método de Runge-Kutta é muito utilizado para a integração de sistemas de EDOs devido sua simplicidade, alta precisão e versatilidade de aplicação (Cunha, 2003).

\section{Metodologia}

No software OCTAVE, foi desenvolvido a modelagem para a obtenção do etanol a partir dos balanços de massa para cada componente, envolvendo três biorreatores. A modelagem foi desenvolvida com a simulação em ambiente computacional que possibilita o estudo aprofundado do projeto de reatores e cinético para a obtenção do etanol. É esperado que com três biorreatores tenham uma maior eficiência na obtenção do produto. Foi realizada a modelagem do sistema de produção de etanol utilizando o modelo matemático de Tosetto. Este método leva em consideração 
os aspectos ligados a quantidade de substratos e a presença de inibidores. A Equação 1 representa o modelo de Tosetto.

$$
\mu=\mu_{\max }\left(\frac{\mathrm{s}}{\mathrm{k}_{\mathrm{S}}+\mathrm{S}+\mathrm{S}^{2} / \mathrm{k}_{\mathrm{i}}}\right)\left(1-\frac{\mathrm{P}}{\mathrm{P}_{\max }}\right)^{\mathrm{n}}
$$

Onde:

$\mu \rightarrow$ taxa de crescimento de microrganismos

$\mu_{\max } \rightarrow$ taxa de máxima crescimento de microrganismos

$\mathrm{S} \rightarrow$ substrato

$\mathrm{k}_{\mathrm{s}} \rightarrow$ constante de substrato

$\mathrm{k}_{\mathrm{i}} \rightarrow$ constante de inibição

$\mathrm{P} \rightarrow$ concentração de produto

$\mathrm{P}_{\max } \rightarrow$ concentração máxima de produto

$\mathrm{n} \rightarrow$ constante de ajuste

Condições consideradas pelo modelo de Tosseto:

- Substrato limitante;

- Inibição pelo substrato;

- Inibição de potência do produto;

A Figura 1 mostra o fluxograma do sistema para a obtenção do etanol a partir de três biorreatores.

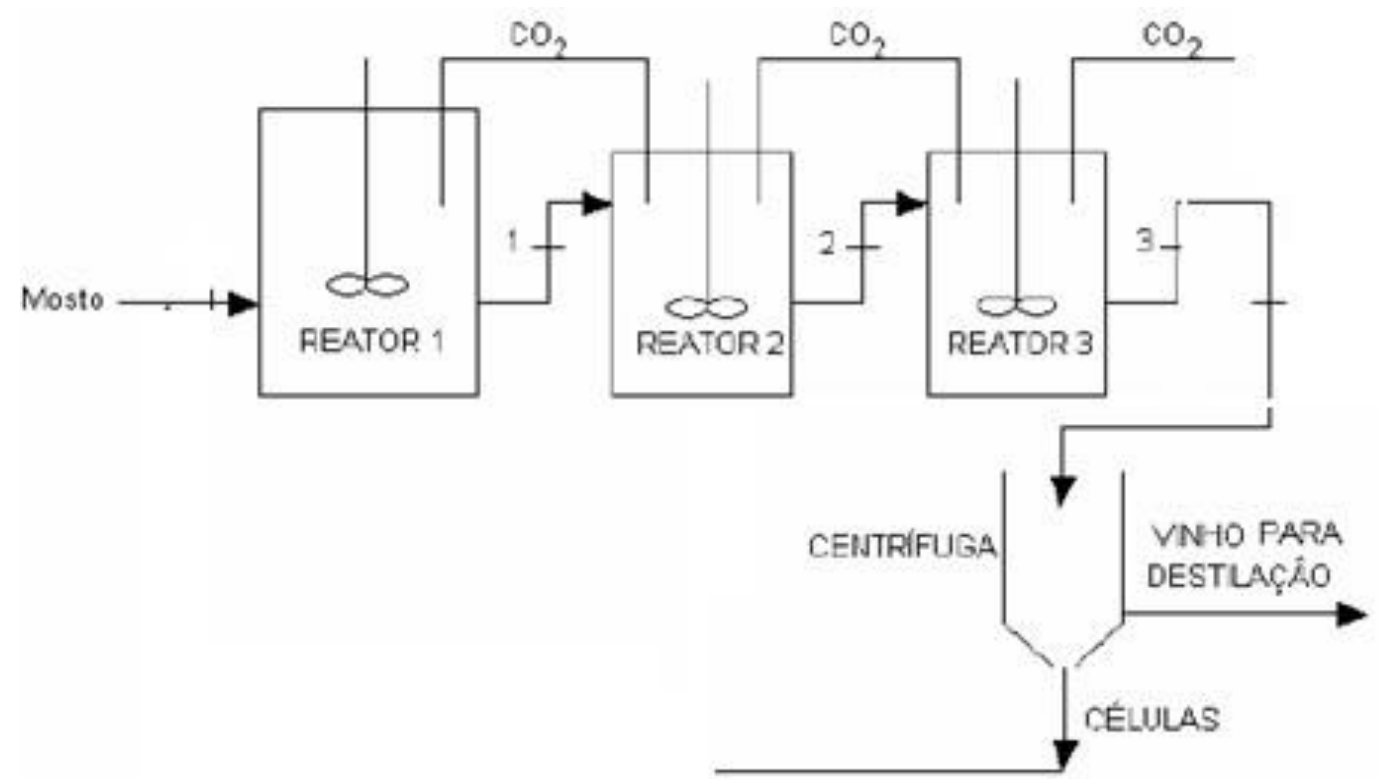

Figura 1- Fluxograma do sistema indicando os três biorreatores em série. 
As Equações Diferenciais 2, 3 e 4 descrevem os balanços de massa genéricos para uma quantidade (i) reatores, que dizem respeito à cinética das reações. A escolha de um modelo que descreve, idealmente, a cinética da reação de produção de etanol é fundamental para a obtenção de resultados fidedignos à literatura (Tosetto, 2002). Sendo assim, o modelo cinético escolhido para o processo foi o de Tosetto (Equação 1). Para iniciar a modelagem considera-se o balanço de massa em regime transiente da quantidade de células, do substrato e do produto, respectivamente, conforme as Equações 2, 3 e 4.

$$
\begin{gathered}
X_{i-1} F_{i-1}-X_{i} F_{i}+\frac{Y_{P} / s}{Y_{X / s}} \mu_{i} X_{i} V_{i}=V_{i} \frac{d X_{i}}{d t} \\
S_{i-1} F_{i-1}-S_{i} F_{i}-\frac{Y_{P} / s}{Y_{X / s}} \mu_{i} X_{i} V_{i}=V_{i} \frac{d S_{i}}{d t} \\
P_{i-1} F_{i-1}-P_{i} F_{i}+\frac{Y_{P} / s}{Y_{X / s}} \mu_{i} X_{i} V_{i}=V_{i} \frac{d P_{i}}{d t}
\end{gathered}
$$

A modelagem matemática dos componentes de interesse a partir das Equações genéricas 2, 3 e 4 para (i) reatores, foi realizada para três biorreatores contínuos em série em regime transiente e foram expressas pelas seguintes Equações diferenciais 5, 8 e 11 para as células, Equações 6, 9 e 12 para o substrato e Equações 7, 10 e 13 para o etanol, respectivamente. O sistema de nove EDO's não-lineares resultante foi integrado numericamente por meio do método de Runge-Kutta, utilizando o software OCTAVE.

- Primeiro biorreator:

Balanço de massa para as células

$\mathrm{X}_{0} \mathrm{~F}_{\mathrm{c}}+\mathrm{X}_{4} \mathrm{~F}_{\mathrm{r}}-\left[\mathrm{X}_{1}\left(\mathrm{~F}_{\mathrm{c}}+\mathrm{F}_{\mathrm{r}}\right)\right]+\frac{Y_{P}}{Y_{\frac{X}{S}}} \mu_{\text {máx }}\left(\frac{\mathrm{s}_{1}}{\mathrm{k}_{\mathrm{S}}+\mathrm{S}_{1}+\mathrm{S}_{1}{ }^{2} / \mathrm{k}_{\mathrm{i}}}\right)\left(1-\frac{\mathrm{P}_{1}}{\mathrm{P}_{\text {máx }}}\right)^{\mathrm{n}} \mathrm{X}_{1} \mathrm{~V}_{1}=\mathrm{V}_{1} \frac{\mathrm{dX}_{1}}{\mathrm{dt}}$

Balanço de massa para o substrato

$\mathrm{S}_{0} \mathrm{~F}_{\mathrm{S}}+\mathrm{S}_{4} \mathrm{~F}_{\mathrm{r}}-\mathrm{S}\left(\mathrm{F}_{2 \mathrm{~S}}+\mathrm{F}_{\mathrm{r}}\right)-\frac{Y_{P}}{Y_{\frac{X}{S}}} \mu_{\text {máx }}\left(\frac{\mathrm{S}_{1}}{\mathrm{k}_{\mathrm{S}}+\mathrm{S}_{1}+\mathrm{S}_{1}{ }^{2} / \mathrm{k}_{\mathrm{i}}}\right)\left(1-\frac{\mathrm{P}_{1}}{\mathrm{P}_{\text {máx }}}\right)^{\mathrm{n}} \mathrm{X}_{1} \mathrm{~V}_{1}=\mathrm{V}_{1} \frac{\mathrm{dS}_{1}}{\mathrm{dt}}$

Balanço de massa para o etanol

$$
\mathrm{P}_{0} \mathrm{~F}_{\mathrm{p}}+\mathrm{P}_{4} \mathrm{~F}_{\mathrm{r}}-\mathrm{P}_{1}\left(\mathrm{~F}_{\mathrm{p}}+\mathrm{F}_{\mathrm{r}}\right)+\frac{Y_{P}}{Y_{\frac{X}{S}}} \mu_{\text {máx }}\left(\frac{\mathrm{s}_{1}}{\mathrm{k}_{\mathrm{S}}+\mathrm{S}_{1}+\mathrm{C}_{1}{ }^{2} / \mathrm{k}_{\mathrm{i}}}\right)\left(1-\frac{\mathrm{P}_{1}}{\mathrm{P}_{\text {máx }}}\right)^{\mathrm{n}} \mathrm{X}_{1} \mathrm{~V}_{1}=\mathrm{V}_{1} \frac{\mathrm{dP} 1}{\mathrm{dt}}
$$


- Segundo biorreator:

Balanço de massa para as células

$$
\mathrm{X}_{1}\left(\mathrm{~F}_{\mathrm{c}}+\mathrm{F}_{\mathrm{r}}\right)-\mathrm{X}_{2}\left(\mathrm{~F}_{\mathrm{c}}+\mathrm{F}_{\mathrm{r}}\right)+\frac{Y_{P}}{Y_{\frac{X}{S}}} \mu_{\text {máx }}\left(\frac{\mathrm{S}_{2}}{\mathrm{k}_{\mathrm{S}}+\mathrm{S}_{2}+\mathrm{S}^{2} / \mathrm{k}_{\mathrm{i}}}\right)\left(1-\frac{\mathrm{P}_{2}}{\mathrm{P}_{\text {máx }}}\right)^{\mathrm{n}} \mathrm{X}_{2} \mathrm{~V}_{2}=\mathrm{V}_{2} \frac{\mathrm{dX}_{2}}{\mathrm{dt}}
$$

Balanço de massa para o substrato

$$
\mathrm{SF}_{\mathrm{z}}+\mathrm{S}_{2}\left(\mathrm{~F}_{\mathrm{s}}+\mathrm{F}_{\mathrm{r}}\right)-\mathrm{S}\left(\mathrm{F}_{3}+\mathrm{F}_{\mathrm{s}}+\mathrm{F}_{\mathrm{z}}\right)-\frac{Y_{P}}{Y_{\frac{X}{S}}} \mu_{\text {máx }}\left(\frac{\mathrm{S}_{2}}{\mathrm{k}_{\mathrm{S}}+\mathrm{S}_{2}+\mathrm{S}^{2}{ }^{2} / \mathrm{k}_{\mathrm{i}}}\right)\left(1-\frac{\mathrm{P}_{2}}{\mathrm{P}_{\text {máx }}}\right)^{\mathrm{n}} \mathrm{X}_{2} \mathrm{~V}_{2}=\mathrm{V}_{2} \frac{\mathrm{dS}_{2}}{\mathrm{dt}}
$$

Balanço de massa para o etanol

$$
\mathrm{P}_{1}\left(\mathrm{~F}_{\mathrm{p}}+\mathrm{F}_{\mathrm{r}}\right)-\mathrm{P}_{2}\left(\mathrm{~F}_{\mathrm{p}}+\mathrm{F}_{\mathrm{r}}\right)+\frac{Y_{P}}{Y_{\frac{X}{S}}} \mu_{\text {máx }}\left(\frac{\mathrm{S}_{2}}{\mathrm{k}_{\mathrm{S}}+\mathrm{S}_{2}+\mathrm{S}_{2}{ }^{2} / \mathrm{k}_{\mathrm{i}}}\right)\left(1-\frac{\mathrm{P}_{2}}{\mathrm{P}_{\text {máx }}}\right)^{\mathrm{n}} \mathrm{X}_{2} \mathrm{~V}_{2}=\mathrm{V}_{2} \frac{\mathrm{dP}_{2}}{\mathrm{dt}}
$$

- Terceiro biorreator:

Balanço de massa para as células

$$
\mathrm{X}_{2}\left(\mathrm{~F}_{\mathrm{c}}+\mathrm{F}_{\mathrm{r}}\right)+\mathrm{X}_{3}\left(\mathrm{~F}_{\mathrm{c}}+\mathrm{F}_{\mathrm{r}}\right)+\frac{Y_{P}}{Y_{\frac{X}{S}}} \mu_{\text {máx }}\left(\frac{\mathrm{S}_{3}}{\mathrm{k}_{\mathrm{S}}+\mathrm{S}_{3}+\mathrm{S}_{3}{ }^{2} / \mathrm{k}_{\mathrm{i}}}\right)\left(1-\frac{\mathrm{P}_{3}}{\mathrm{P}_{\text {máx }}}\right)^{\mathrm{n}} \mathrm{X}_{3} \mathrm{~V}_{3}=\mathrm{V}_{3} \frac{\mathrm{d} X_{3}}{\mathrm{dt}}
$$

Balanço de massa para o substrato

$$
\mathrm{SF}_{\mathrm{z}}+\mathrm{S}_{2}\left(\mathrm{~F}_{\mathrm{s}}+\mathrm{F}_{\mathrm{r}}\right)-\mathrm{S}_{3}\left(\mathrm{~F}_{3}+\mathrm{F}_{\mathrm{s}}+\mathrm{F}_{\mathrm{Z}}\right)-\frac{Y_{P}}{Y_{\frac{X}{S}}} \mu_{\text {máx }}\left(\frac{\mathrm{S}_{3}}{\mathrm{k}_{\mathrm{S}}+\mathrm{S}_{3}+\mathrm{S}^{2} / \mathrm{k}_{\mathrm{i}}}\right)\left(1-\frac{\mathrm{P}_{3}}{\mathrm{P}_{\text {máx }}}\right)^{\mathrm{n}} \mathrm{X}_{3} \mathrm{~V}_{3}=\mathrm{V}_{3} \frac{\mathrm{dS}_{3}}{\mathrm{dt}}
$$

Balanço de massa para o etanol

$$
\mathrm{P}_{2}\left(\mathrm{~F}_{\mathrm{p}}+\mathrm{F}_{\mathrm{r}}\right)+\mathrm{P}_{3}\left(\mathrm{~F}_{\mathrm{p}}+\mathrm{F}_{\mathrm{r}}\right)+\frac{Y_{P}}{Y_{\frac{X}{S}}} \mu_{\text {máx }}\left(\frac{\mathrm{S}_{3}}{\mathrm{k}_{\mathrm{S}}+\mathrm{S}_{3}+\mathrm{S}^{2}{ }^{2} / \mathrm{k}_{\mathrm{i}}}\right)\left(1-\frac{\mathrm{P}_{3}}{\mathrm{P}_{\text {máx }}}\right)^{\mathrm{n}} \mathrm{X}_{3} \mathrm{~V}_{3}=\mathrm{V}_{3} \frac{\mathrm{dP}_{3}}{\mathrm{dt}}
$$

O software OCTAVE foi utilizado para resolver o sistema de nove equações diferenciais. Nesse caso, considerou-se que não há reciclo no sistema $(F r=0)$. Um programa computacional foi desenvolvido no software OCTAVE para resolução do sistema de equações diferenciais e está descrito na Figura 2, sequência (a), (b), (c), (d) e (e). 


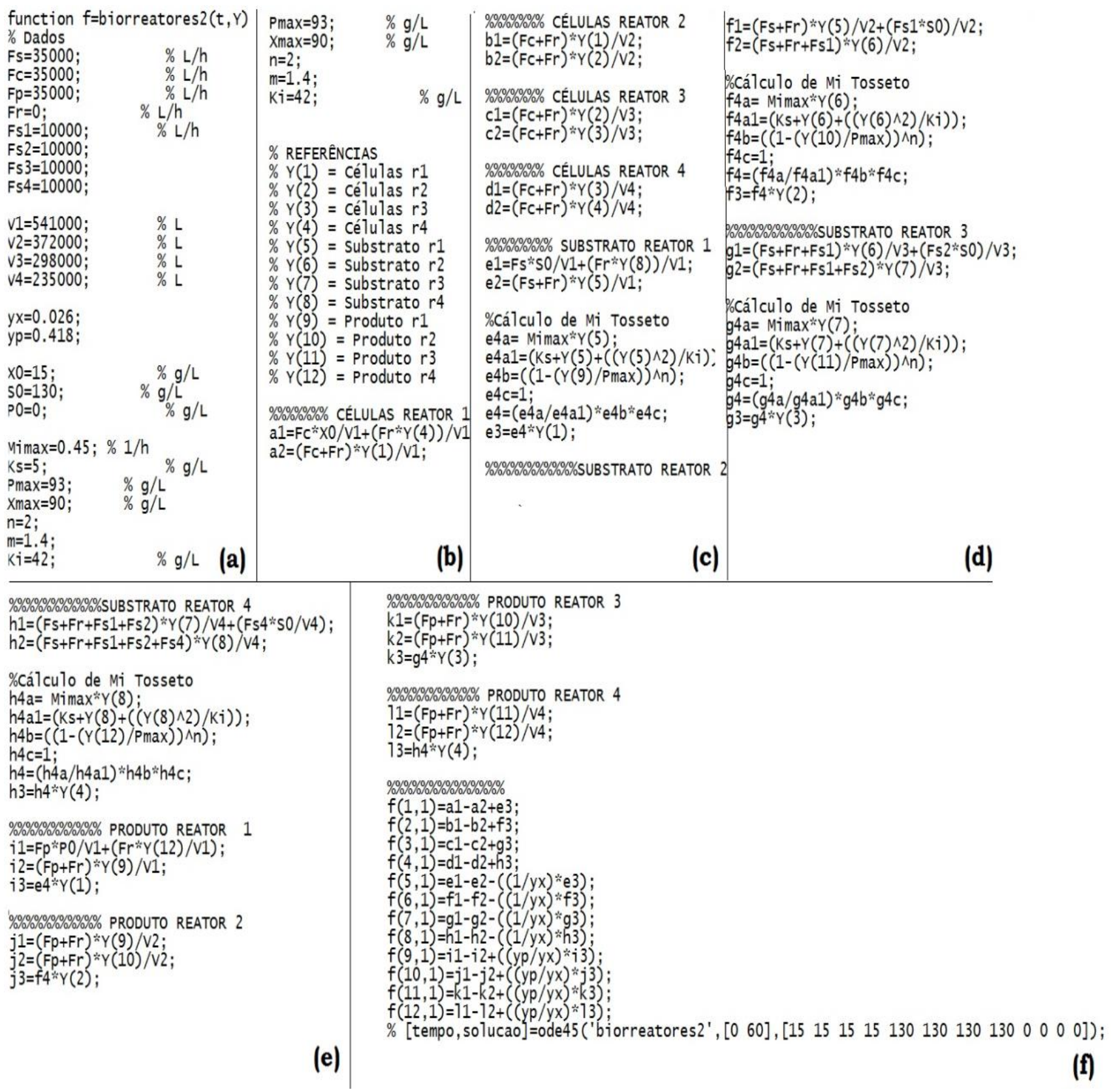

Figura 2- Programa computacional desenvolvido no software OCTAVE para resolução do sistema de equações diferenciais.

\section{Resultados e discussões}

A partir dos resultados obtidos pelo programa computacional da Figura 2 foi gerado o gráfico da simulação da unidade fermentativa composta por três biorreatores ligados em série sem reciclo, conforme a Figura 3. 


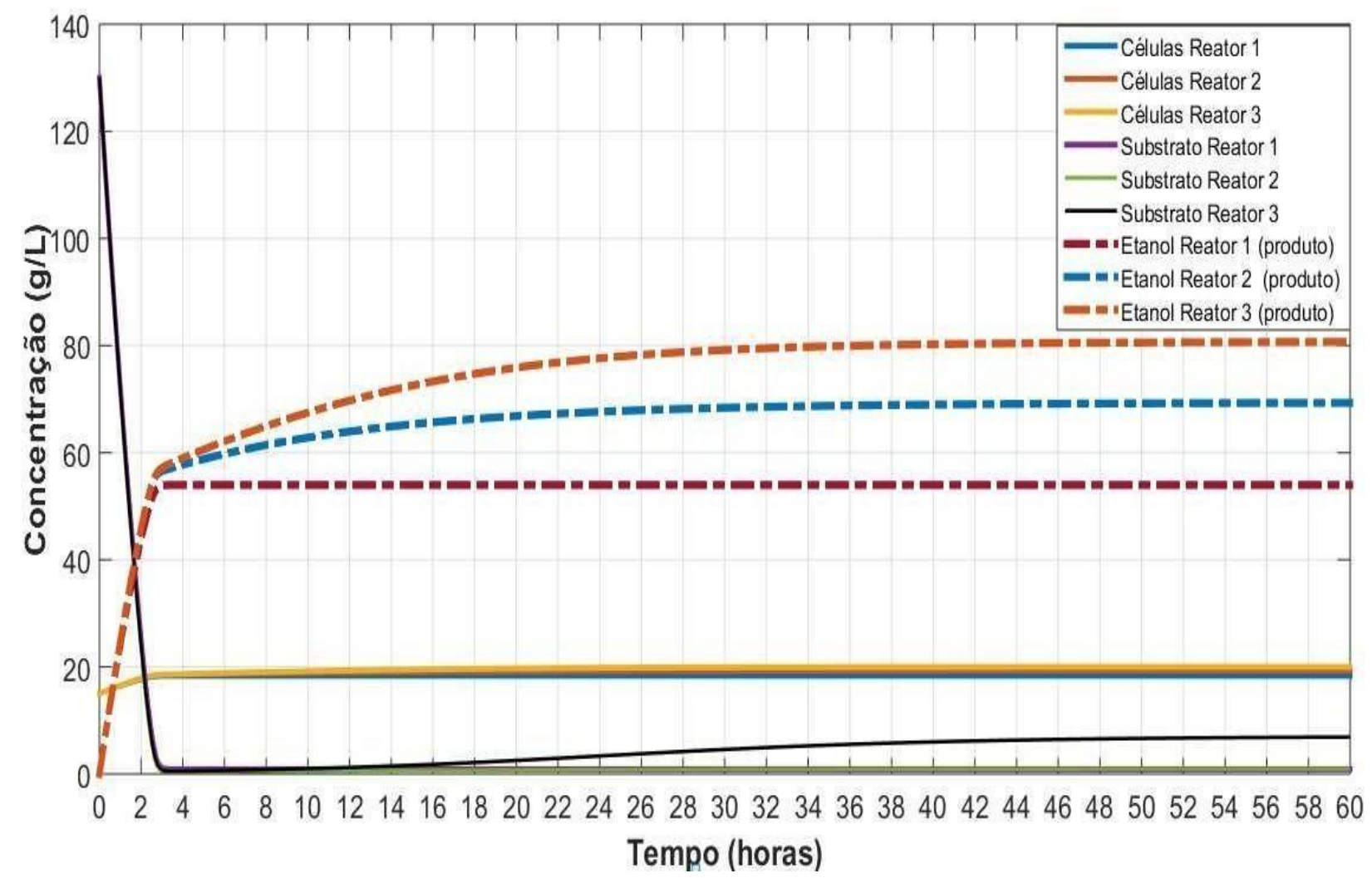

Figura 3- Perfil de consumo de produção de etanol simulado para a unidade fermentativa composta por três biorreatores em série.

A partir da análise da Figura 3 pode-se observar que a concentração de células aumenta significativamente até quatro horas, entrando em regime permanente após este período.

Em relação ao substrato observa-se que sua concentração diminui bruscamente em três horas pelo fato de ser o alimento para o crescimento celular. A partir de dezoito horas observa-se um pequeno crescimento na concentração de substrato no reator três indicando uma inibição pelo substrato.

Em relação aos produtos, a partir da análise da Figura 3, pode-se observar que a concentração inicia em $0 \mathrm{~g} / \mathrm{L}$ e depois aumenta significativamente até as primeiras três horas nos os três biorreatores. Atingindo uma concentração de etanol de $52 \mathrm{~g} / \mathrm{L}$ no reator 1 , uma concentração de etanol $65 \mathrm{~g} / \mathrm{L}$ para o reator 2 e uma concentração de etanol de $80 \mathrm{~g} / \mathrm{L}$ para o reator 3 , em regime permanente.

\section{Conclusão}

Neste trabalho foi possível realizar com êxito as simulações para os três biorreatores contínuos em série pela Equação de Tosetto. Em relação à concentração do produto etanol observou-se na simulação que a operação realizada em três estágios torna o processo mais eficiente ao invés de se utilizar apenas um biorreator. Observou-se nas simulações que para a obtenção do etanol ocorre uma inibição do substrato, não afetando significativamente a produção de etanol. Os resultados das simulações são adequados com o esperado baseado na prática experimental e sugere-se para trabalhos futuros a validação do modelo teórico com dados experimentais obtidos na indústria ou escala piloto, de acordo com Ascendino e Tôrres (2017). 


\section{Referências}

Aguirre, L. A. (2004). Introdução à identificação de sistemas: técnicas lineares e não lineares a sistemas reais. UFMG.

Andrietta, S. R.; Gambaro, V.; Fiorim, F.; Fabro, J. L.; Palmer, C. S.; Maugeri, F. (1994). Fermentação contínua - projeto, controle e aspectos operacionais. STAB.

Ascendino, G. G., Tôrres, L. O. M. (2017). Modelagem de biorreatores contínuos em série com reciclo para a produção de etanol e estudo de caso de usina de álcool. Dissertação de Mestrado, Universidade Federal de São João Del-Rei, Ouro Branco, MG, Brasil.

Cunha, M. C. C. (2003). Métodos Numéricos. Unicamp.

Lima, U. A.; Basso, L. C.; Amorim, H. V. (2001). Biotecnologia Industrial: Processos Fermentativos e Enzimáticos. Biotecnologia Industrial. Edgard Blucher.

Silva, J. L. (2012). Modelagem e simulação de reatores autoclave para produção de PEBD. Dissertação de Mestrado, Universidade Federal do Rio Grande do Sul, Porto Alegre, RS, Brasil.

Souza, G. S. (2016). Estudo da fermentação alcoólica em um biorreator de leito fixo em sistema contínuo com células de Saccharomyces cerevisiae imobilizadas em alginato cálcio revestido com quitosana. Dissertação de Mestrado, Universidade Estadual de Campinas, Campinas, SP, Brasil.

Tosetto, G. (2002). Influência da matéria prima no comportamento cinético de levedura na produção de etanol. Dissertação de Mestrado, Universidade Estadual de Campinas, Campinas, SP, Brasil. 